\title{
Reduction in oral corticosteroid use in patients receiving omalizumab for allergic asthma in the real-world setting
}

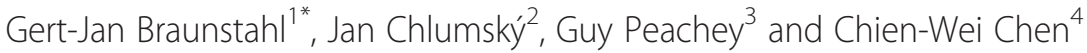

\begin{abstract}
Background: Oral corticosteroids (OCS) are commonly administered in patients with severe persistent allergic asthma. Despite their efficacy, they are associated with a wide variety of adverse events. The eXpeRience registry was set up to investigate real-world outcomes among patients receiving omalizumab for the treatment of uncontrolled allergic asthma. Here, we present the effect of omalizumab treatment on OCS use.

Methods: eXpeRience was a 2-year, multinational, non-interventional, observational registry of patients receiving omalizumab for uncontrolled allergic asthma. OCS use (proportion of patients on maintenance OCS, mean total daily OCS dose and change in status of OCS therapy) was assessed at baseline, 16 weeks, and 8, 12, 18, and 24 months after the initiation of omalizumab. Response to omalizumab was assessed using the physician's Global Evaluation of Treatment Effectiveness (GETE) at approximately Week 16. Safety data were also recorded.
\end{abstract}

Results: A total of 943 patients (mean age, 45 years; female, 64.9\%) were enrolled in the registry, 263 of whom were receiving maintenance OCS at baseline. The proportion of patients taking maintenance OCS was markedly lower at Months 12 (16.1\%) and 24 (14.2\%) than at baseline (28.6\%; intent-to-treat population). GETE status was determined in 915 patients receiving omalizumab: 64.2\% were responders (excellent or good response), 30.7\% were non-responders (moderate, poor or worsening response); $5.1 \%$ had no assessment. The frequency of serious adverse events was comparable to that seen in controlled trials of omalizumab.

Conclusions: Omalizumab use is associated with an OCS-sparing effect in patients with uncontrolled persistent allergic asthma in the real-world setting.

Keywords: Anti-immunoglobulin E, Oral corticosteroid use, Omalizumab, Registry, Uncontrolled persistent allergic asthma

\section{Background}

Patients with allergic asthma are often inadequately controlled despite treatment with high-dose inhaled corticosteroids (ICS) and long-acting $\beta_{2}$-agonists (LABA) [1,2]. Oral corticosteroids (OCS) are commonly administered to suppress airway inflammation and improve asthma control in these patients; however, their long-term use is associated with significant adverse effects, such as diabetes, osteoporosis and cataract formation, placing a major burden on patients and healthcare resources [3-6].

\footnotetext{
*Correspondence: g.braunstahl@sfg.nl

'Department of Pulmonary Medicine, Sint Franciscus Gasthuis, Kleiweg 500, 3045 PM, Rotterdam, The Netherlands

Full list of author information is available at the end of the article
}

Interventions that allow OCS treatment to be reduced or withdrawn completely are likely to benefit patients receiving these agents for the treatment of asthma.

Omalizumab, a humanized anti-immunoglobulin E (IgE) monoclonal antibody, is approved for the treatment of patients with uncontrolled moderate-to-severe (US) or severe (EU) persistent allergic (IgE-mediated) asthma $[7,8]$. Omalizumab has been shown to reduce asthma exacerbations and hospital visits, as well as corticosteroid use, in patients with allergic asthma [9-11]. Omalizumab has also been shown to have a direct OCS-sparing effect in a 32week randomized, open-label study in adolescents and adults (12-75 years) with severe asthma [12], as well as in 
a 16-week uncontrolled therapeutic trial in children (median age 12 years) [13].

eXpeRience was an international registry initiated to evaluate outcomes in patients receiving omalizumab for uncontrolled persistent allergic asthma in 'real-world' clinical practice. The primary results, published previously $[14,15]$, showed that omalizumab was associated with improvements in clinical outcomes such as asthma exacerbations and objective measures of asthma control. Here, we evaluate the real-world effect of omalizumab treatment on the use of OCS over a 2-year period.

\section{Methods}

eXpeRience was a multinational, non-interventional, observational registry established to collect data on the real-world effectiveness and safety of omalizumab therapy during routine clinical practice in patients with uncontrolled persistent allergic (IgE-mediated) asthma. The registry design has been published previously [15].

Briefly, the registry included male and female patients with uncontrolled persistent allergic asthma who had commenced omalizumab treatment within the previous 15 weeks. Patients from 14 countries in Europe, America and Asia were enrolled, and were followed for up to 2 years after initiation of omalizumab. After entry into the registry, data were collected prospectively at approximately 16 weeks and at 8,12, 18 and 24 months after initiation of omalizumab treatment, with a minimum requirement of two data collections per year.

Treatment and follow-up of patients was at the discretion of the treating physician, according to local medical practice and label/reimbursement guidelines. The registry design and amendments were reviewed by independent ethics committees or institutional review boards at each participating centre, as required.

\section{Registry assessments}

Data on OCS use were collected at each pre-determined time-point. The variables evaluated included: proportion of patients receiving OCS as maintenance therapy; total daily OCS dose; change from baseline in OCS dose; number of patients in whom OCS therapy was stopped, reduced (without stopping), or increased as compared with baseline; time to reduction in OCS dose or stopping OCS therapy. Data on ICS use were also collected at each time-point, including: total daily ICS dose; change from baseline in ICS dose; number of patients in whom ICS therapy was stopped, reduced (without stopping), or increased as compared with baseline.

Response to omalizumab was assessed using the physician's Global Evaluation of Treatment Effectiveness (GETE) at approximately Week 16 after the initiation of treatment. OCS use among omalizumab responders (i.e. those with an "excellent" or "good" response by GETE) and non-responders (i.e. "moderate" or "poor" response, or "worsening" asthma) was evaluated. OCS doses were converted to prednisolone equivalents $(1 \mathrm{mg}$ prednisone $=$ $1 \mathrm{mg}$ prednisolone; $1 \mathrm{mg}$ methylprednisolone $=1.25 \mathrm{mg}$ prednisolone).

Safety was assessed by recording the nature and frequency of serious adverse events (SAEs) that occurred during the registry, which were followed until resolution.

\section{Statistical analysis}

All efficacy analyses reported are based on the intent-totreat (ITT) population, consisting of all randomized patients who had at least one post-baseline efficacy assessment. All safety analyses are based on the safety population, which included all patients who received at least one dose of omalizumab and had at least one post-baseline safety assessment.

Statistical analyses were mainly descriptive. Summary statistics describing change from baseline in OCS dose, reduction or cessation of OCS treatment, and time to reduction were calculated for all patients receiving maintenance OCS therapy at baseline, and for GETE-defined responders and non-responders.

\section{Results}

\section{Patient disposition and baseline characteristics}

A total of 943 patients were included in the eXpeRience registry. Of these patients, 694 (73.6\%) completed the registry and $157(16.6 \%)$ discontinued; status was unknown for 92 (9.8\%). The most common reasons for discontinuation were loss to follow-up $(n=52 ; 5.5 \%)$ and withdrawal of consent $(n=27 ; 2.9 \%)$ [14]. Demographic and clinical characteristics of the patients receiving OCS at baseline were comparable with the overall population (Table 1; [14]). The ITT population included 916 patients and the safety population included 925 patients. Of the 263 patients receiving OCS at baseline, the ITT population included 246 patients and the safety population included 263 patients.

\section{OCS use}

The most commonly used OCS was prednisone $(\mathrm{n}=131$ at baseline; $49.8 \%$ of all patients receiving OCS). The proportion of patients receiving OCS was lower at Months 12 (16.1\%) and 24 (14.2\%) than at baseline (28.6\%) (Figure 1). The mean total daily OCS dose (prednisolone equivalent) decreased between baseline (15.5 mg) and Month 12 (7.7 mg), and continued to decrease between Months 12 and 24 (5.8 mg) (Figure 2).

Among patients receiving OCS at baseline, there was a reduction in or discontinuation of OCS treatment in 57.1\% and $69.0 \%$ of patients at Months 12 and 24, respectively (Figure 3). The mean (SD) time to reduction or discontinuation of OCS was 198.5 (114.29) days and 291.2 (210.86) 
Table 1 Baseline demographics and clinical characteristics of patients in the eXpeRience registry (safety population)

\begin{tabular}{|c|c|c|}
\hline Variable & $\begin{array}{l}\text { Patients on OCS at baseline } \\
\qquad(n=263)\end{array}$ & $\begin{array}{l}\text { Overall population } \\
\qquad(\mathrm{n}=925)\end{array}$ \\
\hline Mean age, years (SD) & $46.0(13.3)$ & $45.0(15.0)$ \\
\hline Female, n (\%) & $169(64.3)$ & $600(64.9)$ \\
\hline \multicolumn{3}{|l|}{ Race, $n(\%)$} \\
\hline Caucasian & $246(93.5)$ & $855(92.4)$ \\
\hline Others & $17(6.5)$ & $70(7.6)$ \\
\hline Mean duration of allergic asthma, years (SD) & $20.3(13.6)[n=261]$ & $19.4(13.6)$ \\
\hline Positive skin-prick test/RAST for perennial aeroallergens, $n(\%)$ & $232(88.2)$ & $816(88.2)^{\dagger}$ \\
\hline History of seasonal allergy, $\mathrm{n}(\%)$ & $177(67.3)$ & $587(63.5)^{\dagger}$ \\
\hline \multicolumn{3}{|l|}{ Smoking history, n (\%) } \\
\hline Never smoked & $202(76.8)$ & $719(77.7)^{\ddagger}$ \\
\hline Ex-smoker & $52(19.8)$ & $173(18.7)$ \\
\hline Current smoker & $9(3.4)$ & $30(3.2)$ \\
\hline \multicolumn{3}{|l|}{ Asthma clinical symptoms, $\mathrm{n}(\%)$} \\
\hline Daytime asthma symptoms & $245(93.2)$ & $838(90.6)$ \\
\hline Limitations of activities & $239(90.9)$ & $795(85.9)$ \\
\hline Nocturnal symptoms/awakenings & $218(82.9)$ & $737(79.7)$ \\
\hline \multicolumn{3}{|l|}{ Asthma control (investigator assessment), $\mathrm{n}(\%)$} \\
\hline Controlled & $3(1.1)$ & $13(1.4)^{\dagger}$ \\
\hline Partly controlled & $42(16.0)$ & $215(23.2)$ \\
\hline Uncontrolled & $218(82.9)$ & $693(74.9)$ \\
\hline
\end{tabular}

${ }^{\dagger}$ Data missing for one patient; ${ }^{\ddagger}$ Data missing for three patients.

RAST, radioallergosorbent test; SD, standard deviation.

days, assessed at Months 12 and 24, respectively. Five patients (2.6\%) at Month 12 and four patients $(2.4 \%)$ at Month 24 had an increase in OCS dose.

\section{OCS use according to response}

Of the 915 patients assessed using the GETE, 64.2\% were responders (excellent, 11.4\%; good, 52.8\%) and 30.7\% were

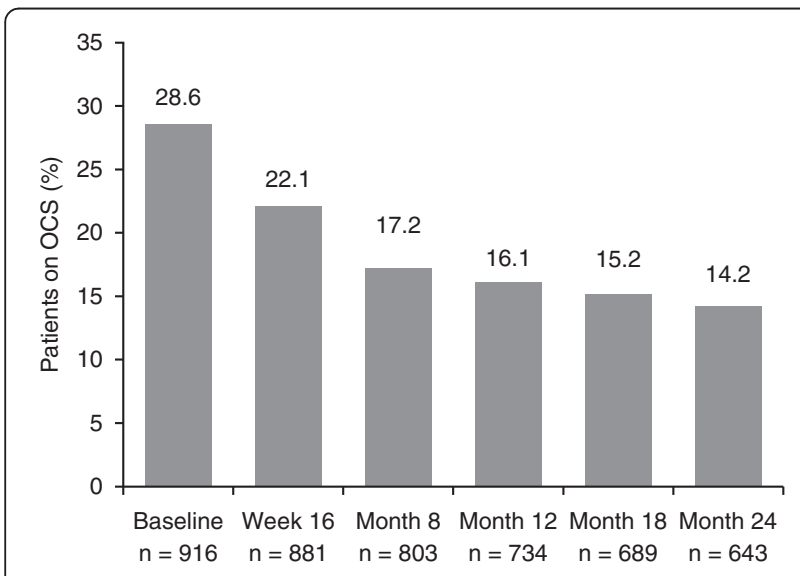

Figure 1 Proportion of patients on maintenance OCS. $n=$ Number of evaluable patients at each time point. OCS, oral corticosteroids. non-responders (moderate, 23.4\%; poor, 6.8\%; worsening of asthma, $0.5 \%$ ) based on GETE status; $5.1 \%$ had no assessment. Following the GETE assessment, the vast majority of responders (98.1\%) and 'moderate' nonresponders $(96.3 \%)$ continued with treatment.

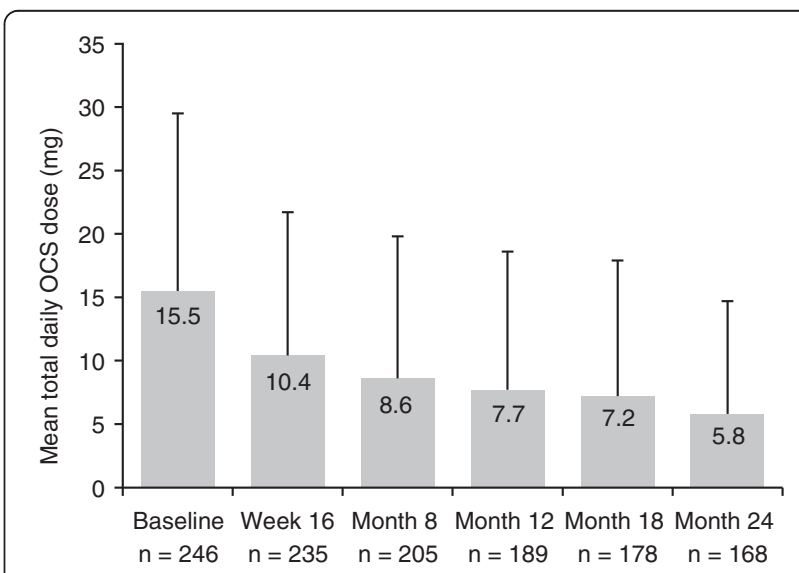

Figure 2 Mean total daily OCS dose (prednisolone equivalent) (ITT population). $n=$ number of evaluable patients at each time-point. OCS, oral corticosteroids; SD, standard deviation; ITT, intent-to-treat. Error bars represent SD. Post-baseline data include doses of zero for patients no longer receiving OCS. 


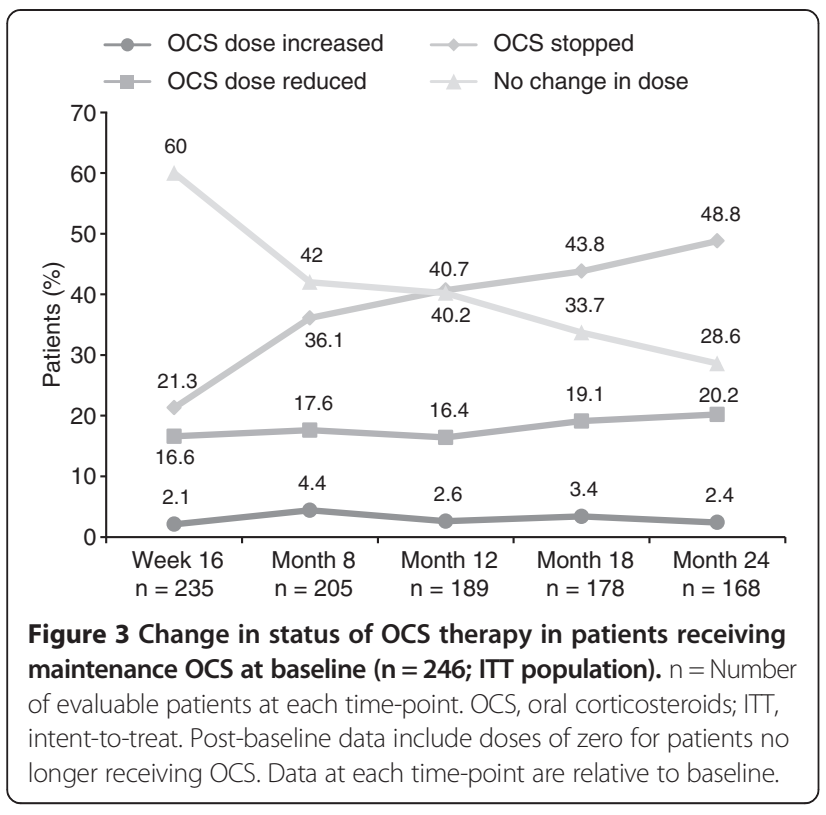

Among responders, the proportion of patients on maintenance OCS treatment decreased from $28.1 \%$ at baseline to $13.8 \%$ at Month 12 and $12.1 \%$ at Month 24 . Mean (SD) total daily dose (prednisolone equivalent) decreased from 15.5 (14.63) $\mathrm{mg}$ at baseline to 6.5 (10.13) $\mathrm{mg}$ at Month 12, and 5.1 (8.94) $\mathrm{mg}$ at Month 24.

Among GETE non-responders, the proportion of patients on maintenance OCS treatment decreased from $28.5 \%$ at baseline to $20.9 \%$ at Month 12 and $17.9 \%$ at Month 24. Mean (SD) total daily dose (prednisolone equivalent) decreased from 14.7 (10.92) $\mathrm{mg}$ at baseline to $11.3(12.85) \mathrm{mg}$ at Month 12 , and 8.1 (8.99) $\mathrm{mg}$ at Month 24.

\section{ICS use}

At baseline, 895 of 916 patients (98\%) were receiving ICS maintenance therapy. Among patients who provided ICS information, 173 of 705 (24.5\%) and 182 of 613 (29.7\%) had stopped or reduced ICS use at Months 12 and 24, respectively. The majority of patients did not change their ICS dose (495 [70.2\%] at Month 12 and 389 [63.5\%] at Month 24), while 37 patients (5.2\%) and 42 patients (6.9\%) had an increase in dose at Months 12 and 24, respectively. Mean (SD) total daily ICS doses (beclomethasone equivalent) decreased from 1675 (947) $\mu \mathrm{g}$ at baseline to 1461 (950) at Month 12 and 1381 (961) at Month 24. Mean (SD) percentage reductions in ICS dose from baseline were $9.6 \%(49.8)$ at Month 12 and $11.8 \%$ (57.1) at Month 24.

\section{ICS use according to response}

Among GETE responders, the mean (SD) ICS dose decreased from baseline by 266 (759) $\mu \mathrm{g}$ at Month 12 and
313 (819) $\mu \mathrm{g}$ at Month 24, with mean (SD) percentage reductions of 12.9 (41.8) and 14.8 (47.4), respectively. Among the responders, 133 of 487 (27.3\%) and 137 of $435(31.5 \%)$ had stopped or reduced ICS use at Months 12 and 24 , respectively.

Among GETE non-responders, the mean (SD) ICS dose decreased from baseline by 134 (847) $\mu \mathrm{g}$ at Month 12 and 246 (1122) $\mu$ g at Month 24, with mean (SD) percentage reductions of 2.8 (65.9) and 4.8 (77.9), respectively. Among the non-responders, 40 of 200 (20.0\%) and 44 of $164(26.8 \%)$ had stopped or reduced ICS use at Months 12 and 24, respectively.

\section{Safety}

Detailed safety findings from the registry have been published previously [14]. Briefly, a total of 64 patients (6.9\%) reported 150 SAEs. Of these, 25 SAEs (16.7\%) were suspected to be related to omalizumab. The most common SAE was asthma $(\mathrm{n}=32,3.5 \%)$, followed by dyspnoea and pneumonia (both $n=7,0.8 \%$ ). Nine deaths occurred during the registry; none were suspected to be related to omalizumab (causes of death have been described previously [14]).

\section{Discussion}

In this registry, omalizumab was associated with a reduction in maintenance OCS use in patients with uncontrolled persistent allergic asthma over a 2-year treatment period in a real-world setting. Approximately half of the patients on maintenance OCS at baseline were able to stop or reduce their OCS dose. In addition, there were also reductions in maintenance ICS use over the 2 years of the study. We believe that the observed reductions in OCS and ICS use reflect improved asthma control during treatment with omalizumab, and are likely to be associated with a reduction in the risk of steroid-related adverse effects. Reductions in OCS use and mean total daily OCS dose were greater in patients classified as responders to omalizumab treatment than in non-responders. Nevertheless, a reduction in OCS use and mean total daily OCS dose was seen in non-responders, possibly due to the fact that the definition of non-responders included patients with a moderate response to omalizumab (as well as those with a poor or worsening response). This is highlighted by the vast majority of moderate 'non-responders' continuing with treatment, following the GETE assessment.

Corticosteroids are widely prescribed to treat inflammatory conditions, including asthma, for which they are often the mainstay of treatment [5]. Patients with uncontrolled severe asthma may require long-term maintenance therapy with OCS. However, such use is associated with serious long-term adverse effects such as hypothalamic-pituitary-adrenal axis suppression, impaired glucose tolerance and diabetes, osteoporosis, hypertension, 
and cataract formation. There is therefore a need for therapies that improve outcomes, have acceptable safety and tolerability profiles, while allowing reductions in OCS use [12].

Despite the observed reductions in OCS use, patients enrolled in the eXpeRience registry had fewer clinically significant asthma exacerbations after 12 or 24 months' treatment with omalizumab (annualized mean 1.0 and 0.6 , respectively) compared with pre-treatment values (annualized mean 4.9) [14]. Relatedly, omalizumab was also associated with reductions in healthcare utilization (hospitalizations, emergency room visits and unscheduled doctor visits), from 6.2 during the 12-month pretreatment period to 1.0 per year at Month 12 and 0.5 per year at Month 24 [14]. Annualized numbers of days of absence from work and school due to asthma were also lower at Month 12 (3.5 and 1.6 days, respectively) and Month 24 (1.0 and 1.9 days, respectively) than during the 12-month pre-treatment period (26.4 and 20.7 days, respectively) [14].

Our findings are in agreement with those of other real-life studies of omalizumab. A pooled analysis of data from French and German patients $(n=346)$ with severe persistent allergic asthma showed that omalizumab treatment for at least 16 weeks was associated with a reduction or discontinuation of OCS in $50 \%$ of patients receiving OCS at baseline $(n=84 / 166)$ [16]. The mean reduction in daily OCS dose from baseline was $74.3 \%$ [16]. An additional French historic-prospective study showed that $48.1 \%$ of patients reduced or discontinued maintenance OCS over a period of $\geq 5$ months of omalizumab treatment [17].

Decreased use of OCS subsequent to treatment with omalizumab has also been shown in observational studies conducted in Italy [18], Belgium [19], Israel [20] and the United Kingdom [21]. These studies enrolled between 22 and 142 patients, who were followed up for between 16 and 52 weeks. Between 20\% and 70\% of patients taking OCS at baseline were able to stop or reduce treatment, and showed meaningful reductions in exacerbations rates.

The results of this analysis indicate that a significant proportion of patients on omalizumab therapy were classed as responders by physician's GETE at Week 16 . These results are consistent with omalizumab clinical trials. In an early randomized controlled study, 53\% of omalizumab-treated patients had an excellent/good response to omalizumab, compared with $33 \%$ for placebo [22]; in a more recent study, $72.8 \%$ in the omalizumab plus optimized asthma therapy (OAT) group responded, compared to $31.2 \%$ for OAT alone [23]. However, not all responses to omalizumab are achieved within the first 16 weeks of therapy, with some patients taking longer to respond [23].
Bousquet et al. demonstrated that the physician's GETE at Week 16 is an effective predictor of longerterm outcomes, including exacerbation rates, overall asthma control and unscheduled medical interventions [24]. Subsequently, Bousquet et al. also showed that the majority of patients classified as responders or nonresponders at Week 16 have the same classification at Week 32 [23]. Consistent with the findings of Bousquet et al. [23,24], the present study also indicated that asthma control (as reflected in OCS use) is improved to a greater extent among responders to omalizumab, compared with non-responders, and that these improvements (and the differences between responders and nonresponders) persist between Week 16 and 2 years.

Most of the observational studies conducted with omalizumab support a reduction in maintenance OCS dose to improve asthma management. However, in rare cases, patients receiving omalizumab may present with systemic hypereosinophilic syndrome or allergic eosinophilic granulomatous vasculitis (Churg-Strauss syndrome) [25], and these events are usually, but not always, associated with a reduction in OCS dose. We did not observe any cases of either hypereosinophilic syndrome or ChurgStrauss syndrome in the eXpeRience registry, but we believe that clinicians attempting OCS reduction or withdrawal in patients with allergic asthma should be aware of this.

Despite our positive findings, it is important to recognize the limitations of observational studies, namely the lack of a control group and the open-label design.

\section{Conclusions}

In conclusion, this 2-year, international and observational registry, conducted in a real-life setting, confirms that omalizumab is associated with OCS-sparing effects in patients with uncontrolled persistent allergic (IgEmediated) asthma.

\section{Abbreviations}

OCS: Oral corticosteroids; GETE: Global Evaluation of Treatment Effectiveness; ICS: Inhaled corticosteroids; LABA: Long-acting $\beta_{2}$-agonists; IgE: Immunoglobulin E; SAEs: Serious adverse events; ITT: Intent-to-treat; OAT: Optimized asthma therapy.

\section{Competing interests}

G-JB has received grant/research support for consultations and/or speaking at conferences from Novartis, GSK, AstraZeneca, and MSD.

$\mathrm{JC}$ has received lecture fees from Novartis, and has been an investigator in studies sponsored by several other pharmaceutical companies. C-WC and GP are Novartis employees.

\section{Authors' contributions}

G-JB has contributed to the enrolment of patients, data capture and processing and has reviewed and commented various drafts of the manuscript. JC has contributed to enrolment of patients and has reviewed and commented various drafts of the manuscript. GP is a clinical lead accountable for the conduct and reporting of the registry; oversight of all operational aspects of the registry (e.g. patient enrolment; document management, etc.); scientific review of registry data, and lead author of the 
clinical study reports (interim and final). C-WC has contributed to data analysis. All authors read and approved the final manuscript.

\section{Acknowledgements}

The registry was sponsored by Novartis Pharma AG. The authors were assisted in the preparation of the manuscript by Dr. Madhavi Dokku (Novartis). Writing support was funded by the registry sponsor.

\section{List of Independent Ethics Committees or Institutional Review Boards} - Argentina, Buenos Aires: Comite Independiente De Etics Para Ensayos En Farmacologia Clinica • Bulgaria, Sofia: Ethics Committee for Multicenter Trials. Canada, Alberta: University of Calgary Conjoint Health Research Ethics Board. Canada, British Columbia: University of British Columbia, Office of Research Ethics• Canada, Nova Scotia: Capital Health Research Ethics Board; University Health Network• Canada, Ontario: IRB Services• Canada, Québec: Centre de Recherche du CHUM; Comité d'éthique du Centre de santé et de services sociauc du Nord de Lansudière; Research Ethics Board Montreal Children's Hospital; Centre de santé et de services sociaux de Laval, Cité de la Santé de Laval. Cyprus, Nicosia: Cyprus National Bioethics Committee. Czech Republic, Prague: Ethics Committee of the AlFP. Hungary, Budapest: Egészségügyi Tudományos Tanács Tudományos és Kutatásetikai Bizottság• Netherlands: N/A (EC approval not required in Netherlands). Philippines, Manila: Our Lady of Lourdes Hospital Ethics and Review Committee • Portugal, Almada: Comissão de ética do Hospital Garcia de Orta• Portugal, Amadora: Comissão de ética do Hospital Fernando Fonseca. Portugal, Coimbra: Comissão de ética do CHC. Portugal, Colvilhã: Comissão de ética do Centro Hospitalar Cova da Beira. Portugal, Évora: Comissão de ética do HESE. Portugal, Faro: Comissão de ética Hospital de Faro• Portugal, Guarda: Comissão de ética da Unidade Local de Saúde da Guarda. Portugal, Lisboa: Comissão de ética do Centro Hospitalar Lisboa Norte; Comissão de ética do Centro Hospitalar de Lisboa Ocidental• Portugal, Penafiel: Comissão de ética do Centro Hospitalar do Tâmega e Sousa • Portugal, Portalegre: Comissão de ética da Unidade Local de Saúde do Norte Alentejano• Portugal, Porto: Comissão de ética do Hospital São João• Portugal, Santarém: Comissão de ética Hospital Distrital de Santarém. Portugal, Torres Vedras: Comissão de ética do Centro Hospitalar de Torres Vedras• Portugal, Vila Nova de Gaia: Comissão de ética do Centro Hospitalar Gaia-Espinho• Russia: N/A (EC approval not required in Russia). Slovakia, Bratislava: Bratislavsky Samosprávny Kraj• Slovenia, Ljubljana: Komisija Republike Slovenije za Medicinsko Etiko• Spain, Córdoba: Comite Etico de Investigación Clinica. Spain, Mallorca: Comite Etico de Investigacion Clinica de las Illes Baleares, Coselleria de Salut y Consum• Spain, San BartoloméOrihuela: Comite Etico de Investigación Clínica• Spain, Santiago de Compostela: Comite Etico de Investigacion Clinica de Galicia• Spain, Sevilla: CAEC- Comite Coordinador de Etica de la Investigacion Biomedica de Andalucia• Spain, Valencia: Dirección general de Farmacia y Productos Sanitarios; Conselleria de Sanitat, Dirección general de Farmacia y Productos Sanitarios. Spain, VitoriaGasteiz: Comite Etico de Investigacion Clinica- hospital Virgen de las Nieves; CEIC-E, Direccion de Farmacia del Departamento de Sanidad del Gobierno Vasco• Taiwan, Taichung: National Cheng Kung University Hospital Institutional Review Board • Taiwan, Tainan City: Taichung Veterans General Hospital Institutional Review Board • Taiwan, Taipei: National Taiwan University Hospital Research Ethics Committee; Chang Gung Memorial Hospital Institutional Review Board.

\section{Author details \\ 'Department of Pulmonary Medicine, Sint Franciscus Gasthuis, Kleiweg 500, 3045 PM, Rotterdam, The Netherlands. ${ }^{2}$ Department of Pulmonary Disease, Thomayer Hospital, Charles University, Prague, Czech Republic. ${ }^{3}$ Novartis Pharmaceuticals UK Limited, Horsham, West Sussex, UK. ${ }^{4}$ Novartis Pharmaceuticals Corporation, East Hanover, NJ, USA.}

Received: 01 August 2013 Accepted: 20 November 2013 Published: 04 December 2013

\section{References}

1. Hanania NA, Alpan O, Hamilos DL, Condemi JJ, Reyes-Rivera I, Zhu J, Rosen KE, Eisner MD, Wong DA, Busse W: Omalizumab in severe allergic asthma inadequately controlled with standard therapy: a randomized trial. Ann Intern Med 2011, 154:573-582.
2. Humbert M, Beasley R, Ayres J, Slavin R, Hébert J, Bousquet J, Beeh KM Ramos S, Canonica GW, Hedgecock S, Fox H, Blogg M, Surrey K: Benefits of omalizumab as add-on therapy in patients with severe persistent asthma who are inadequately controlled despite best available therapy (GINA 2002 step 4 treatment): INNOVATE. Allergy 2005, 60:309-316.

3. Curtis JR, Westfall AO, Allison J, Bijlsma JW, Freeman A, George V, Kovac SH, Spettell CM, Saag KG: Population-based assessment of adverse events associated with long-term glucocorticoid use. Arthritis Rheum 2006, 55:420-426

4. Fardet L, Flahault A, Kettaneh A, Tiev KP, Généreau T, Tolédano C, Lebbé C, Cabane J: Corticosteroid-induced clinical adverse events: frequency, risk factors and patient's opinion. Br J Dermatol 2007, 157:142-148.

5. Manson SC, Brown RE, Cerulli A, Vidaurre CF: The cumulative burden of oral corticosteroid side effects and the economic implications of steroid use. Respir Med 2009, 103:975-994.

6. Walsh LJ, Wong CA, Oborne J, Cooper S, Lewis SA, Pringle M, Hubbard R, Tattersfield AE: Adverse effects of oral corticosteroids in relation to dose in patients with lung disease. Thorax 2001, 56:279-284.

7. EMA Xolair SMPC. http://www.ema.europa.eu/docs/en_GB/document library/EPAR_Product_Information/human/000606/WC500057298.pdf.

8. US Food and Drug Administration: Omalizumab (Xolair ${ }^{\circledR}$ ) prescribing information. http://www.fda.gov/Drugs/DrugSafety/PostmarketDrugSafetylnformationfor PatientsandProviders/ucm126456.htm.

9. Holgate ST, Chuchalin AG, Hebert J, Lötvall J, Persson GB, Chung KF, Bousquet J, Kerstjens HA, Fox H, Thirlwell J, Cioppa GD: Omalizumab 011 International Study Group: efficacy and safety of a recombinant antiimmunoglobulin $\mathrm{E}$ antibody (omalizumab) in severe allergic asthma. Clin Exp Allergy 2004, 34:632-638.

10. Lafeuille MH, Dean J, Zhang J, Duh MS, Gorsh B, Lefebvre P: Impact of omalizumab on emergency-department visits, hospitalizations, and corticosteroid use among patients with uncontrolled asthma. Ann Allergy Asthma Immunol 2012, 109:59-64

11. Solèr M, Matz J, Townley R, Buhl R, O'Brien J, Fox H, Thirlwell J, Gupta N, Della Cioppa G: The anti-IgE antibody omalizumab reduces exacerbations and steroid requirement in allergic asthmatics. Eur Respir J 2001, 18:254-261.

12. Siergiejko Z, Świebocka E, Smith N, Peckitt C, Leo J, Peachey G, Maykut R: Oral corticosteroid sparing with omalizumab in severe allergic (IgE-mediated) asthma patients. Curr Med Res Opin 2011, 27:2223-2228.

13. Brodlie M, McKean MC, Moss S, Spencer DA: The oral corticosteroidsparing effect of omalizumab in children with severe asthma. Arch Dis Child 2012, 97:604-609.

14. Braunstahl G-J, Chen C-W, Maykut R, Georgiou P, Peachey G, Bruce J: The eXpeRience registry: the 'real-world' effectiveness of omalizumab in allergic asthma. Resp Med 2013, 107:1141-1151.

15. Braunstahl G-J, Leo J, Thirlwell J, Peachey G, Maykut R: Uncontrolled persistent allergic asthma in practice: eXpeRience registry baseline characteristics. Curr Med Res Opin 2011, 27:761-767.

16. Molimard M, Buhl R, Niven R, Le Gros V, Thielen A, Thirlwell J, Maykut R, Peachey G: Omalizumab reduces oral corticosteroid use in patients with severe allergic asthma: real-life data. Respir Med 2010, 104:1381-1385.

17. Molimard M, de Blay F, Didier A, Le Gros V: Effectiveness of omalizumab (Xolair) in the first patients treated in real-life practice in France. Respir Med 2008, 102:71-76.

18. Cazzola M, Camiciottoli G, Bonavia M, Gulotta C, Ravazzi A, Alessandrini A, Caiaffa MF, Berra A, Schino P, Di Napoli PL, Maselli R, Pelaia G, Bucchioni E, Paggiaro PL, Macchia L: Italian real-life experience of omalizumab. Respir Med 2010, 104:1410-1416.

19. Brusselle G, Michils A, Louis R, Dupont L, Van de Maele B, Delobbe A, Pilette C, Lee CS, Gurdain S, Vancayzeele S, Lecomte P, Hermans C, MacDonald K, Song M, Abraham I: "Real-life" effectiveness of omalizumab in patients with severe persistent allergic asthma: The PERSIST study. Respir Med 2009, 103:1633-1642.

20. Rottem M: Omalizumab reduces corticosteroid use in patients with severe allergic asthma: real-life experience in Israel. J Asthma 2012, 49:78-82.

21. Niven R: A UK survey of oral corticosteroid use in patients treated with omalizumab [abstract]. Thorax 2007, 62(3):A98. P91.

22. Busse W, Corren J, Lanier BQ, McAlary M, Fowler-Taylor A, Cioppa GD, van As A, Gupta N: Omalizumab, anti-lgE recombinant humanized monoclonal antibody, for the treatment of severe allergic asthma. J Allergy Clin Immunol 2001, 108:184-190. 
23. Bousquet J, Siergiejko Z, Świebocka E, Humbert M, Rabe KF, Smith N, Leo J, Peckitt C, Maykut R, Peachey G: Persistency of response to omalizumab therapy in severe allergic (IgE-mediated) asthma. Allergy 2011, 66:671-678.

24. Bousquet J, Rabe K, Humbert M, Chung KF, Berger W, Fox H, Ayre G, Chen H, Thomas K, Blogg M, Holgate S: Predicting and evaluating response to omalizumab in patients with severe allergic asthma. Respir Med 2007, 101:1483-1492.

25. Wechsler ME, Wong DA, Miller MK, Lawrence-Miyasaki L: Churg-strauss syndrome in patients treated with omalizumab. Chest 2009, 136:507-518.

doi:10.1186/1710-1492-9-47

Cite this article as: Braunstahl et al:: Reduction in oral corticosteroid use in patients receiving omalizumab for allergic asthma in the real-world setting. Allergy, Asthma \& Clinical Immunology 2013 9:47.

\section{Submit your next manuscript to BioMed Central and take full advantage of:}

- Convenient online submission

- Thorough peer review

- No space constraints or color figure charges

- Immediate publication on acceptance

- Inclusion in PubMed, CAS, Scopus and Google Scholar

- Research which is freely available for redistribution 